\title{
Population pharmacokinetics of pyrimethamine and sulfadoxine in children with congenital toxoplasmosis
}

\author{
T. Trenque, N. Simon, ${ }^{1}$ I. Villena, ${ }^{2}$ C. Chemla, ${ }^{2}$ C. Quereux, ${ }^{3}$ B. Leroux, ${ }^{3}$ R. Jaussaud, ${ }^{3}$ G. Rémy, ${ }^{3}$ D. Dupouy, ${ }^{3}$ H. Millart, \\ J.-M. Pinon' ${ }^{2}$ \& S. Urien ${ }^{4}$ \\ Laboratoire de Pharmacologie-Toxicologie, CHU, Reims, ${ }^{1}$ Faculté de Médecine, Université de Marseille, Marseille, ${ }^{2}$ Laboratoire de \\ Parasitologie, IFR53, Université de Reims Champagne-Ardenne, CHU, Reims, ${ }^{3}$ Reims Toxoplasmosis Group, CHU, Reims, and ${ }^{4}$ Centre René \\ Huguenin, Saint Cloud, France
}

\section{Correspondence \\ Thierry Trenque MD, PhD, Laboratoire de Pharmacologie- Toxicologie, Centre Hospitalier Universitaire, 45 Rue Cognacq-Jay, 51092 Reims Cedex, France. \\ Tel: + 33326787780 \\ Fax: + 33326788456 \\ E-mail: ttrenque@chu-reims.fr}

\section{Keywords}

children, congenital toxoplasmosis, pharmacokinetics, population, pyrimethamine, sulfadoxine

\section{Received}

15 July 2003

Accepted

9 December 2003

\begin{abstract}
Aims
To develop a population pharmacokinetic model for pyrimethamine (PYR) and sulfadoxine (SDX) in children with congenital toxoplasmosis.

Methods

Children were treated with PYR (1.25 $\mathrm{mg} \mathrm{kg}^{-1}$ ) and SDX $\left(25 \mathrm{mg} \mathrm{kg}^{-1}\right)$ (Fansidar ${ }^{\circledR}$ ) plus folinic acid (Lederfoline ${ }^{\circledR} 5 \mathrm{mg}$ ). Plasma concentrations, available from a therapeutic drug monitoring database, were determined by high-performance liquid chromatogrphy. Population pharmacokinetic analysis was performed using a nonlinear mixed effects model.
\end{abstract}

\section{Results}

Eighty-nine children, aged 1 week to 14 years and weighing 2.9-59 kg, were available for evaluation. Both PYR and SDX concentration-time profiles were best described by a one-compartment open model. Volume of plasma distribution $(V)$ and clearance $(\mathrm{CL})$ were significantly related to body weight (BW) using an allometric function. Typical $\mathrm{CL}$ and $V$ estimates (95\% confidence interval), for a child weighing $11 \mathrm{~kg}$ were $5.50(5.28,5.73) \mid$ day $^{-1}$ and $36(33,39) \mid$ for PYR and $0.26(0.25,0.27) \mid$ day $^{-1}$ and $2.1(1.9,2.3)$ I for SDX. For BW between 3.5 and $60 \mathrm{~kg}$, plasma half-lives were predicted to vary from 4.0 to 5.2 days for PYR, and from 5.0 to 7.5 days for SDX.

\section{Conclusion}

This study indicated that body weight influences PYR and SDX pharmacokinetics in children. To optimize PYR/SDX combination treatment in congenital toxoplasmosis, short dosing intervals in very young low-wight children are probably appropriate.

\section{Introduction}

The combination of pyrimethamine (PYR) and sulfadoxine (SDX) (Fansidar ${ }^{\circledR}$ ) is frequently used for the treatment of Toxoplasma gondii infection (toxoplasmosis) $[1,2]$, which is generally asymptomatic. However, when acquired during pregnancy, it can lead to congenital toxoplasmosis, which in the absence of treatment can cause mental retardation, and toxoplasmic retinochoroidites with a risk of blindness [3]. These compli- cations can be prevented by early treatment of the child with PYR-SDX combinations [4-6].

Our centre uses a continuous regimen combining PYR and SDX (Fansidar ${ }^{\circledR}, 500 \mathrm{mg}$ SDX and $25 \mathrm{mg}$ PYR) [7]. PYR is a synthetic diaminopyridine antagonist of folonic acid, and SDX is an ultralong-acting sulphonamide. PYR inhibits dihydrofolate reductase and SDX inhibits dihydropteroate synthetase. This regimen significantly decreases the likelihood of the devel- 
opment of sequelae in infected children $[8,9]$. The combination of PYR and SDX is also used in the treatment of malaria $[10,11]$.

Information about the pharmacokinetics of these drugs is very limited, particularly in children. The pharmacokinetic profiles of PYR and SDX have been investigated in a limited number of patients $[12,13]$. Maximum plasma concentrations of both drugs are reached within $4 \mathrm{~h}$ after oral administration. The elimination half-lives of PYR and SDX range from 4 to 4.8 days and 7.7 to 10.6 days, respectively. A pharmacokinetic study of PYR has been reported in 37 infants between the ages of 10 days and 1.5 years [14] and a blood pharmacokinetics study of SDX has been reported in 10 children (8-14 years old) [15]. In children between the ages of 10 days and 1.5 years, the elimination halflives of PYR and SDX were found to be 2.2-3.6 days and in children between the ages of 8 and 14 years they were 5.2-10.1 days, respectively.

The pharmacokinetics of drugs are expected to vary greatly in children of different ages. Moreover, adult studies do not necessarily predict pharmacokinetics in children. For these reasons, clinicians treating paediatric patients with Fansidar ${ }^{\circledR}$ have difficulties in adjusting the dose, with respect to both the amount given and the dosing interval. This is reflected in practice by numerous requests for PYR and SDX serum concentrations.

An understanding of the pharmacokinetics of the combination of PYR-SDX in children with congenital toxoplasmosis could be helpful in developing treatment regimens that minimize side-effects and maintain efficacy. Therefore, we conducted a population pharmacokinetic study of PYR and SDX using a therapeutic drug monitoring database established in our laboratory.

\section{Methods}

Patients and treatment

At the beginning of the study (15 years ago), we discussed the protocol with our local ethics committee, who advised that written consent from a parent or guardian was not necessary. In collaboration with clinicians, we created the Reims Toxoplasmosis Group [1, 16], which managed the treatment of children with congenital toxoplasmosis (CT). Eighty-nine children were monitored prospectively by regular clinical and laboratory measurements from birth until 13.9 years old. All had a positive neonatal diagnosis of congenital toxoplasmosis. The PYR $\left(1.25 \mathrm{mg} \mathrm{kg}^{-1}\right)$-SDX $\left(25 \mathrm{mg} \mathrm{kg}^{-1}\right)$ combination (Fansidar ${ }^{\circledR}$ ) plus folinic acid (Lederfoline ${ }^{\circledR}$ $5 \mathrm{mg}$ ) was given three times a month or twice a month. The treatment was started as soon as the diagnosis was confirmed. The children underwent monthly physical and ophthalmic examination for the first 3 months, then every 3 months until the age of 12 months at 16, 24, 28, 32,36 and 48 months, then yearly. An immunological assessment was also performed at these times.

Blood samples were collected for immunological monitoring, and PYR-SDX analysis.

Drug concentration data obtained from 89 children were analysed retrospectively. Information on dosing regimens and PYR and SDX plasma concentrations were obtained from the drug therapeutic monitoring database of the Laboratoire de Pharmacologie (CHU Reims, France). Plasma concentrations were analysed if there was complete information on drug administration, i.e. dosing intervals and amount prescribed. It was necessary to know the time of dosing prior to the plasma concentration determination. For each patient, gender, body weight (BW) and age were recorded. Patients using anticonvulsivant therapy were excluded, because of the risk of drug interactions.

\section{Drug analysis}

SDX and PYR plasma concentrations were measured using high-pressure liquid chromatography (HPLC). Only $150 \mu \mathrm{l}$ of sample were necessary to assay both compounds.

Chromatography was performed using a Varian (Sunnyvale, CA, USA) Model 5000 equipped with a 20$\mu l$ universal loop injector (Valco Instruments, Houston, TX, USA), a variable wavelength Spectroflow 783 UV detector (Kratos, Ramsey, NJ, USA) and a Linseis L600 stripchart recorder (Bioblock Scientifics, Illkirch, France). Separation and analysis were carried out on a reversed-phase system with a C8 column (Lichrospher 100 RP-8, $250 \times 4$ mm i.d., $5 \mu \mathrm{m}$; Merck, Darmstadt, Germany) linked to a $\mathrm{C} 8$ precolumn, $35 \times 4 \mathrm{~mm}$ i.d., packed with Vydac reversed phase material (Varian).

SDX was extracted from $50 \mu \mathrm{l}$ of plasma with methanol and injected directly into the chromatograph. The mobile phase was $0.01 \mathrm{M}$ acetonitrile, $0.02 \mathrm{M}$ tetramethylammonium chloride ( $\mathrm{pH} 2.8)(37 / 63 \mathrm{v} / \mathrm{v})$. The flow rate was $1.2 \mathrm{ml} \mathrm{min}^{-1}$ and the chromatographic system was operated at room temperature. The detection limit of SDX (defined at a signal-to-noise ratio of 2) was $0.5 \mathrm{mg} \mathrm{l}^{-1}$. Within-day coefficients of variation were between 1.36 and $3.11 \%$ and between-day coefficients of variation were between 6.24 and $12.28 \%$.

For PYR, $100 \mu \mathrm{l}$ of plasma were placed in a microtube, and $10 \mu \mathrm{l}$ of sodium hydroxide $(4 \mathrm{M})$ and internal standard $\left(20 \mu \mathrm{l}\right.$ of $500 \mu \mathrm{g} \mathrm{l}^{-1}$ midazolam solution) were added. The solution was stirred for 1 min with $800 \mu$ of ether on a Heidolph Top-Mix shaker (Bioblock Scien- 
tifics) and centrifuged. The organic layer was transferred into a tapered glass tube and evaporated at $40{ }^{\circ} \mathrm{C}$ under dry nitrogen. The residue was dissolved in $50 \mu \mathrm{l}$ of methanol and $20 \mu \mathrm{l}$ were injected into the chromatograph. The mobile phase was $0.01 \mathrm{M}$ acetonitrile, $0.02 \mathrm{M}$ tetramethylammonium chloride $(\mathrm{pH} 3)(37 / 63 \mathrm{v} / \mathrm{v})$. The flow rate was $1.2 \mathrm{ml} \mathrm{min}^{-1}$ and the chromatographic system was operated at room temperature. The eluent was monitored at $240 \mathrm{~nm}$.

The limit of detection of PYR was $5 \mu \mathrm{g} 1^{-1}$ at a signalto-noise ratio of 3. Betwwen-day coefficients of variation $(n=5)$ were $13.4 \%$ at $25 \mu \mathrm{g} \mathrm{l}^{-1}, 14.8 \%$ at $50 \mu \mathrm{g}$ $\mathrm{l}^{-1}, 13.2 \%$ at $100 \mu \mathrm{g} \mathrm{l}^{-1}, 7.6 \%$ at $250 \mu \mathrm{g} \mathrm{l^{-1 }}$ and $7.0 \%$ at $500 \mu \mathrm{g} \mathrm{l}^{-1}$. The within-day coefficient of variation $(n=5)$ at $25 \mu \mathrm{g} \mathrm{l}^{-1}$ was $9.8 \%$, and was below $7 \%$ over the range $50-500 \mu \mathrm{g} \mathrm{l}^{-1}$.

\section{Population pharmacokinetic modelling}

PYR and SDX concentration-time data were analysed using a non-linear mixed effects modelling approach, using the programs MP2 [17] and NONMEM, version V (double precision, level 1.1) [18]. The step-by-step analysis was performed using MP2, and the final population models were also run with NONMEM for confirmation purposes. One- and two-compartment pharmacokinetic open models with first-order absorption and elimination were compared. The available data were not sufficient to estimate a value for $k_{\mathrm{a}}$, and thus this parameter value was fixed at $1.30 \mathrm{~h}^{-1}$ based on a rich-data study of the pharmacokinetics of pyrimethamine and sulfadoxine ( $n=12$ subjects). At different steps in the analysis, the effect of varying $k_{\mathrm{a}}$ was investigated to confirm the validity of the value of $1.30 \mathrm{~h}^{-1}$ used.

A proportional error model with constant coefficient of variation and an additive random effects model were used to describe interpatient and residual variability. A graphical analysis of predicted $v s$. observed (PRED vs. OBS) concentrations was performed to test the value of each model. Comparison between the means of the individual Bayesian (i.e. POSTHOC) parameter estimates and the population estimates was also performed to discriminate between the error models. Between-occasion variability for CL and $V$ was also tested in some children receiving three dosages or more. The influence of each patient covariate on pharmacokinetic parameters was tested systematically via a generalized modelling according to an allometric function. For example, the effect of BW on CL was assessed from the following equation:

$$
\mathrm{CL}=\mathrm{TV}(\mathrm{CL}) \times[\mathrm{BW} / \mathrm{mean}(\mathrm{BW})]^{\theta \mathrm{BW}}
$$

where TV(CL) is the typical value of clearance for a patient with the mean covariate value and $\theta \mathrm{BW}$ is the estimated influential factor for body weight. The effect of gender was tested using the equation

$$
\mathrm{CL}=\mathrm{TV}(\mathrm{CL})\left(1+\theta_{\text {gender }} \mathrm{I}_{\text {gender }}\right)
$$

in which $I_{\text {gender }}$ is the indicator variable $(0=$ male, $1=$ female) and $\theta_{\text {gender }}$ the fraction that changes CL in girls compared with boys.

Because this population included some very young children ( $<6$ months), the covariate analysis also included the following models investigating age-related changes in CL and volume of distribution $(V)$ [18]

$$
\begin{gathered}
\mathrm{CL}=\mathrm{TV}(\mathrm{CL}) \times[\mathrm{BW} / \mathrm{mean}(\mathrm{BW})]^{\theta \mathrm{BW}} \\
\times\left[1-\theta_{1 \mathrm{CL}} \times \mathrm{EXP}\left(-\mathrm{AGE} \times 0.7 / \theta_{2 \mathrm{CL}}\right)\right] \\
\mathrm{V}=\mathrm{TV}(\mathrm{V}) \times[\mathrm{BW} / \mathrm{mean}(\mathrm{BW})]^{\theta \mathrm{BW}}\left[1+\theta_{1 \mathrm{~V}}\right. \\
\left.\times \mathrm{EXP}\left(-\mathrm{AGE} \times 0.7 / \theta_{2 \mathrm{~V}}\right)\right]
\end{gathered}
$$

where $\theta_{1 \mathrm{CL}}$ and $\theta_{1 \mathrm{~V}}$ are the parameters estimating the fractional differences from TV(CL) and TV(V), and $\theta_{2 \mathrm{CL}}$ and $\theta_{2 \mathrm{~V}}$ are the parameters estimating the half-lives of age-related changes in CL and $V$.

Full and reduced models (including one less parameter) were compared by the $\chi^{2}$ test with respect to the difference between their respective objective function values. A decrease of at least $6.635(P<0.01$, one degree of freedom) was required for the addition of a single parameter to the model. The effect of a covariate was considered to have improved the fit if there was a significant decrease in the objective function value of at least 6.635 compared with the basic pharmacokinetic model (with no covariate). An intermediate multivariate model was then obtained including all of the significant covariates. To keep only those covariates making the largest contribution in predicting PYR and SDX pharmacokinetics in a final multivariate model, a change of $10.827(P<0.001$, one degree of freedom) of the objective function was required for the retention of a single parameter during backward stepwise multiple regression analysis. At this step, decrease in the interindividual variability estimate was also considered.

\section{Results}

A total of 89 children ( 35 boys/54 girls), aged 1 week to 14 years and weighing $3-59 \mathrm{~kg}$ were available for pharmacokinetic evaluation. Treatment was never discontinued because of an adverse event. Only one transient skin reaction was observed.

Table 1 summarizes the main pharmacokinetic features of the study. A one-compartment model with firstorder absorption and elimination adequately described the data. Parameters of the final structural model were the plasma clearance (CL), the fixed absorption constant rate $\left(k_{\mathrm{a}}\right)$ and the central volume of distribution $(V)$. CL 
Table 1

Summary of pharmacokinetic data

\begin{tabular}{lllcc}
\hline & PYR & & \multicolumn{2}{c}{ SDX } \\
Item & Median & Range & Median & Range \\
& & & 292 & 3 \\
Number of plasma samples & 312 & $1-13$ & $46.1 \mu \mathrm{g} / \mathrm{ml}$ & $1-12$ \\
Number of plasma samples per patient & 3 & $2.5-678 \mathrm{ng} / \mathrm{ml}$ & 187.5 & $31.25-1000$ \\
Plasma concentration & $95.5 \mathrm{ng} / \mathrm{ml}$ & $3.125-50$ & 15 & $7-15$ \\
Dose (mg) & 9.375 & $7-15$ & & \\
Dosing interval (days) & 15 & & & \\
\hline
\end{tabular}

Table 2

Population pharmacokinetic estimates for PYR and SDX in 89 children aged 1 week to 14 years

\begin{tabular}{|c|c|c|c|c|}
\hline \multirow{2}{*}{$\begin{array}{l}\text { Drug } \\
\text { Parameter }\end{array}$} & \multicolumn{2}{|l|}{ PYR } & \multicolumn{2}{|l|}{ SDX } \\
\hline & estimate & $\mathrm{Cl}$ & estimate & $\mathrm{Cl}$ \\
\hline $\operatorname{TV} . V(I)^{*}$ & 36 & $33-39$ & 2.07 & $1.92-2.31$ \\
\hline TV.CL $\left(\mid \text { day }^{-1}\right)^{*}$ & 5.50 & $5.28-5.73$ & 0.26 & $0.25-0.27$ \\
\hline \multicolumn{5}{|l|}{ Covariate effects } \\
\hline$V, \theta_{\mathrm{BW}}\left(\mathrm{kg}^{-1}\right)$ & 0.60 & $0.49-0.72$ & 0.72 & $0.60-0.86$ \\
\hline $\mathrm{CL}, \theta_{\mathrm{BW}}\left(\mathrm{kg}^{-1}\right)$ & 0.53 & $0.46-0.59$ & 0.64 & $0.58-0.70$ \\
\hline \multicolumn{5}{|l|}{ Interindividual variabilities } \\
\hline$C L(\%)$ & 12.4 & $8.5-17.7$ & 16.3 & $11.7-22.2$ \\
\hline$V(\%)$ & $\mathrm{NE}$ & & $\mathrm{NE}$ & \\
\hline
\end{tabular}

*TV, Typical value, corresponding to a child weighing $11 \mathrm{~kg}$. FFixed parameter. $\neq$ Proportional component. §Absolute (additive) component. NA, Not applicable; NE, not estimated; $V$, central volume of distribution; CL, clearance; $k_{a}$ first-order absorption rate constant.

and $V$ are apparent parameters, including bioavailability $(F), \mathrm{CL} / F$ and $V / F$.

For both PYR and SDX, the proportional and additive components of residual variability were significant. Interindividual variability on $V$ approached zero and exclusion of this parameter had no effect on the objective function value. Between consultation variability for $\mathrm{CL}$ and $V$ was not significant, possibly because there was only one plasma sample at each consultation. The models were not disturbed by attempts to change $k_{\mathrm{a}}$ from 1 to $2 \mathrm{~h}^{-1}$. i.e. Thus, for the two drugs no significant changes in objective function values or in parameter estimates were observed.

In the preliminary screening phase, the covariates that decreased the objective function by more than 7 units were BW and age, both had positive influences on CL and $V$. For both PYR and SDX, the use of $\mathrm{BW}$ instead of age in the allometric equations led to smaller objective functions. Since these covariates were strongly correlated, only BW was retained in the submodelling. The inclusion of BW in the estimation of both $V$ and $\mathrm{CL}$ decreased the objective function by 86 and 165 units for PYR and SDX, respectively. Deletion of the BW increased the objective function by at least 45 units, for both PYR and SDX.

Table 2 summarizes the population parameter estimates. The improvement of the fit from the basic model 
(a)

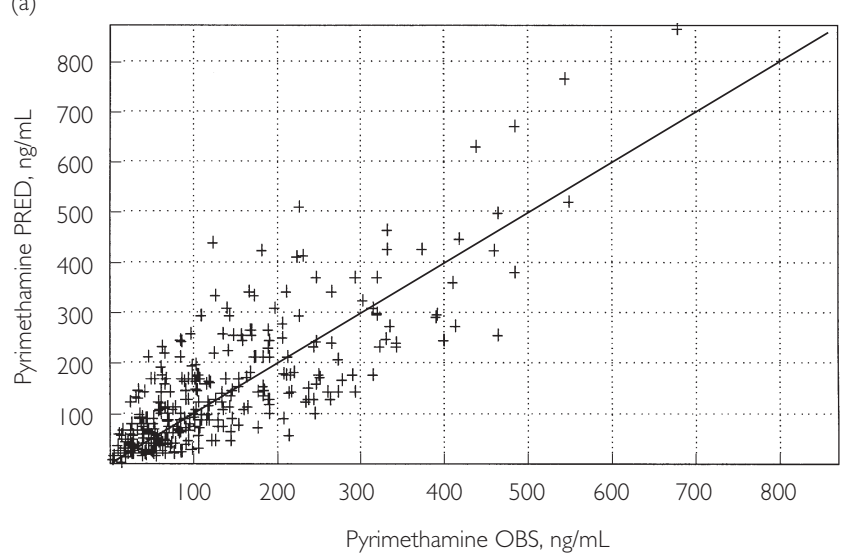

(b)

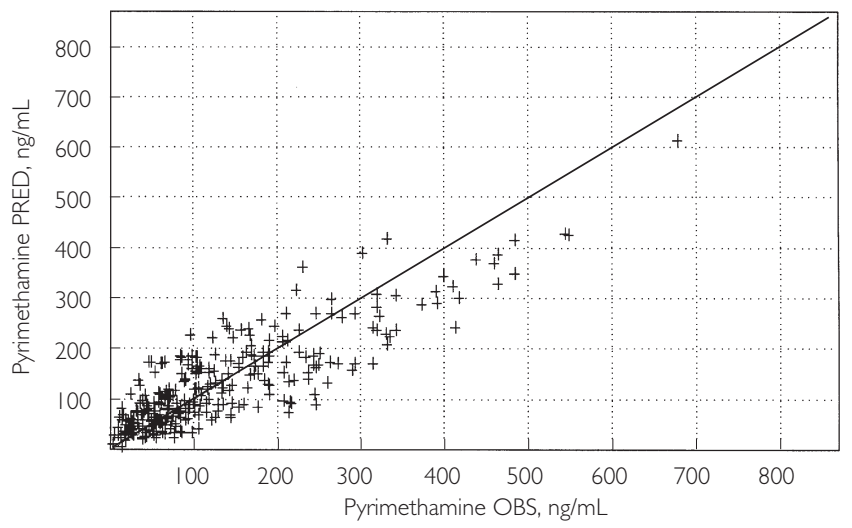

Figure 1

Predicted (PRED) vs. observed (OBS) plasma pyrimethamine concentrations based on the basic, covariate-free, model (a) and the final pharmacokinetic model, including the effect of body weight on $\mathrm{CL}$ and $V$ (b) $(n=89$ children $)$

(without covariate) to the final model is depicted in Figure 1 for PYR and Figure 2 for SDX.

The addition of the age-related change equations was not significant for PYR. However, significance was observed for SDX CL, decreasing the objective function by 24 units, i.e. 12 units per added parameter. The effect on SDX $V$ was not significant. The $\theta_{1 \mathrm{CL}}$ and $\theta_{2 \mathrm{CL}}$ estimates were 0.53 and 0.23 years ( 3 months), respectively, which predict that CL should increase with age, with a half-life of 3 months, to a maximal value of approximately 1 year. Since the decrease in objective function was just above our criterion of 11 units to retain a covariate, the residual plots (OBS-PRED) concentrations $v s$. age were compared between the 'allometric covariate modelling' and the 'allometric covariate modelling with age-related changes'. There was also no improvement in the interindividual variability estimate

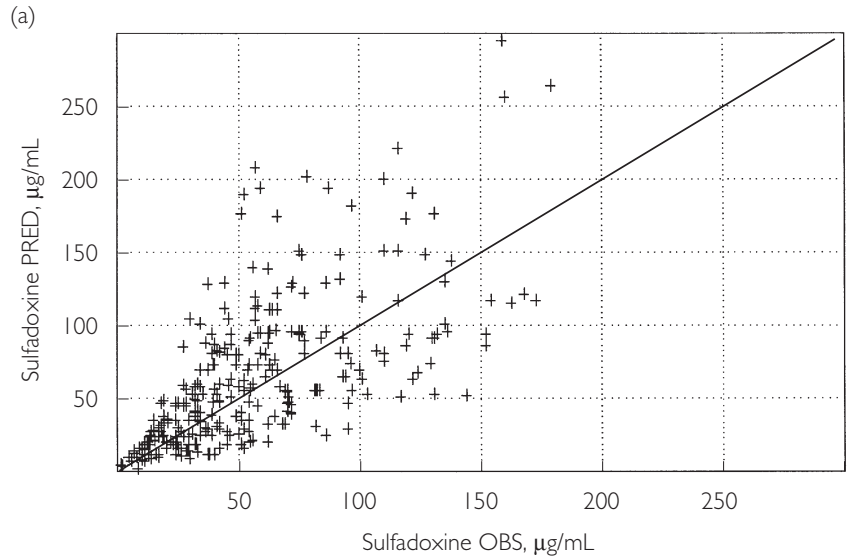

(b)

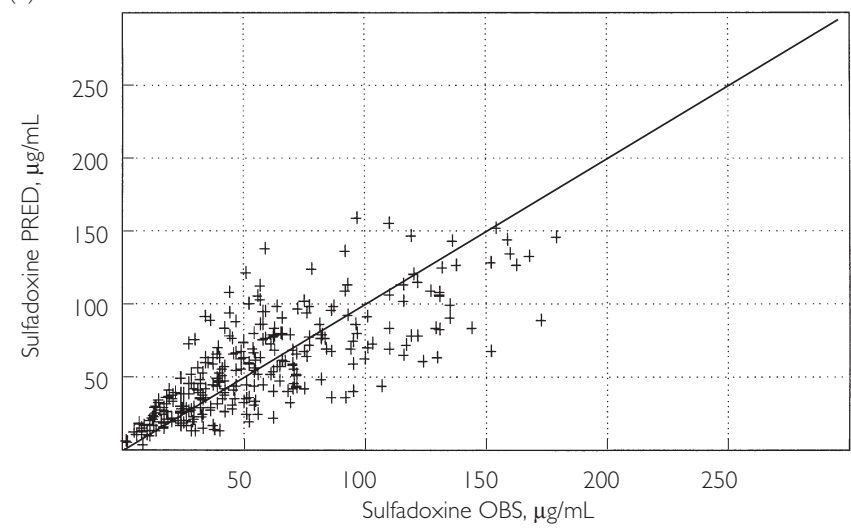

Figure 2

Predicted (PRED) vs. observed (OBS) plasma sulfadoxine concentrations based on the basic, covariate-free, model (a) and the final pharmacokinetic model, including the effect of body weight on $\mathrm{CL}$ and $V$ (b) $(n=89$ children)

of CL. On the basis of these results, and because the other diagnostic plots did not show substantial differences between these two covariate submodelings, we decided to retain the simpler, 'allometric', covariate modelling.

\section{Discussion}

PYR and SDX plasma pharmacokinetics in children were best described by a one-compartment open model with first-order elimination and absorption. Using a classical approach, the PYR and SDX pharmacokinetics in adults were described using a bicompartmental model $[12,13]$. However, this model could not be applied to the present data, since sufficient data in the hours following drug administration were not available, because most of the samples were taken between 3 and several days after administration. 
Body weight and age significantly influenced PYR and SDX plasma pharmacokinetics. The inclusion of BW decreased the associated interindividual variabilities in the CL of the drugs from 16.8 to $12.4 \%$ and from 21.0 to $16.3 \%$, respectively. The choice of both BW and the shape of the relationship between BW and the pharmacokinetic parameters was based on allometric equations or power functions that could be reasonably applied considering the wide range of BW $(3-59 \mathrm{~kg})$ in this population. The typical power exponents for CL and $V$ should be 0.75 and 1.0, respectively [19], which are close to our estimates of $0.53-0.65$ and $0.60-0.76$. The parameters, which are typical pharmacokinetic values and power exponents, were estimated with good accuracy, as shown by their $95 \%$ confidence intervals. In a second step, the possible effect of age was investigated based on a model used for paracetamol pharmacokinetics in children and infants [20]. The influence of age on PYR pharmacokinetics was not found to be significant. For SDX, age significantly influenced only CL, and in the first 6 months of life. However, this effect was considered to be marginal and not retained in the final model.

Figure 3 depicts PYR and SDX half-lives as a function of BW. PYR half-life is predicted to be $4.2-4.5$ days for an infant aged between 15 days and 1.5 years (3.5$11 \mathrm{~kg} \mathrm{BW}$ based on our database). These values for halflives are slightly longer than the 2.2-3.6 days previously reported in similar infants [14]. For SDX, a previous study reported half-lives of between 5.2 and 10.1 days for 8-14-year-old children [15]. This range is similar to our predicted values of 6.2-6.7 days. Clearance estimates of $17.4 \pm 5.30$ and $2.0 \pm 0.31 \mathrm{day}^{-1}$ (or 12.1 and $1.40 \mathrm{ml} \mathrm{min}^{-1}$ ) were reported in young adults for PYR

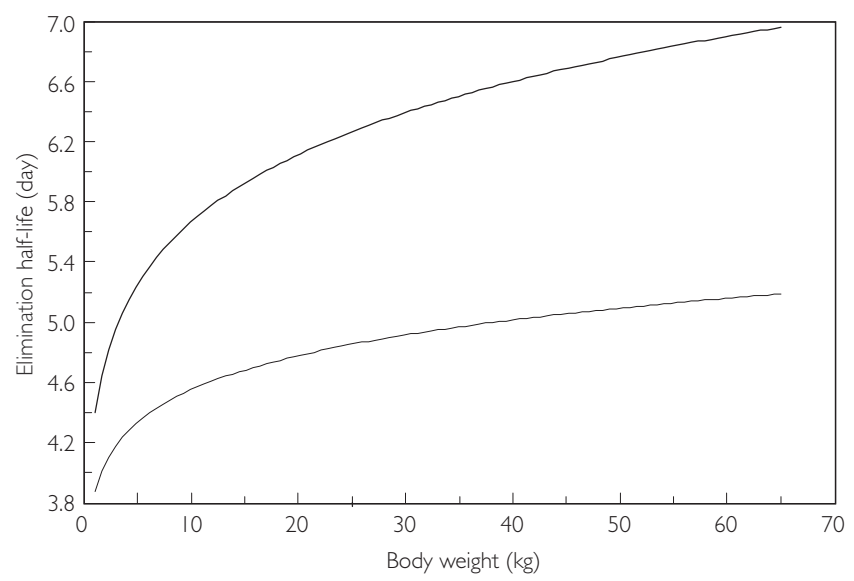

Figure 3

Elimination half-lives of sulfadoxine (upper curve) and pyrimethamine (lower curve) as a function of body weight and SDX, respectively $(n=12)$, given a $60-\mathrm{kg}$ BW the PYR and SDX CL values derived from this study should be 13.5 and 0.81 day $^{-1}$ [12]. Adult half-lives have been reported to be 4.0 to 4.75 days for PYR and 7.66 to 10.6 days for SDX $[12,13]$. These results are in accordance with the higher values of the half-life BW functions of Figure 3.

In conclusion, the present study of a paediatric population with congenital toxoplasmosis treated by a commercial PYR-SDX combination indicates that the time intervals between consecutive Fansidar ${ }^{\circledR}$ administrations should be based on body weight or the age of the child to ensure a minimal continuous drug exposure. Thus, the lower the body weight, the shorter the time interval should be. A time interval between doses of 7 days for children less than 2 years old and 14 days for older children might be appropriate.

\section{References}

1 Villena I, Aubert D, Leroux B et al. Pyrimethamine-sulfadoxine treatment of congenital toxoplasmosis: follow-up of 78 cases between 1980 and 1997. Scand J Infect Dis 1998; 30: 295300

2 Schoondermark-van de Ven E, Vree T, Melchers W, Camps W, Galama J. In vitro effects of sulfadiazine and its metabolites alone and in combination with pyrimethamine on toxoplasma gondii. Antimicrob Agents Chemother 1995; 39: 763-5.

3 Koppe JG, Loewer-Sieger DH, De Roever-Bonnet $\mathrm{H}$. Results of 20 years follow up of congenital toxoplasmosis. Lancet 1986; 1: 254-6.

4 Guerina NG. Congenital infection with Toxoplasma gondii. Pediatr Ann 1994; 23: 138-51.

5 Harris C, Salgo MP, Tanowitz HB, Wittner M. In vitro assessment of antimicrobial agents against Toxoplasma gondii. J Infect Dis 1988; 157: 14-22.

6 Derouin F, Chastang C. Enzyme immunoassay to assess effect of antimicrobial agents on Toxoplasma gondii in tissue culture. Antimicrob Agents Chemother 1988; 32: 303-7.

7 Foulon W, Villena I, Stray-Pedersen B et al. Treatment of toxoplasmosis during pregnancy: a multicenter study of impact on fetal transmission and children's sequelae at age 1 year. Am J Obstet Gynecol 1999; 180: 410-5.

8 Daffos F, Forestier F, Capella-Pavlovsky $M$ et al. Prenatal management of 746 pregnancies at risk for congenital toxoplasmosis. N Engl J Med 1988; 318: 271-5.

9 Hohlfeld P, Daffos F, Thulliez P et al. Fetal toxoplasmosis: outcome of pregnancy and infant follow-up after in utero treatment. J Pediatr 1989; 115: 765-9.

10 Dorsey G, Njama D, Kamya MR et al. Sulfadoxine/pyrimethamine alone or with amodiaquine or artesunate for treatment of uncomplicated malaria: a longitudinal randomized trial. Lancet 2002; 360: 2031-8. 
11 Lindberg A, Bergqvist Y, Rombo L. Accidental overdose of pyrimethamine and sulfadoxine for malaria prophylaxis without adverse effects. Eur J Clin Pharmacol 1986; 31: 253-4.

12 Mansor SM, Navaratnam V, Mohamad M et al. Single dose kinetic study of the triple combination mefloquine/sulphadoxine/ pyrimethamine (Fansimef) in healthy male volunteers. Br J Clin Pharmacol 1989; 27: 381-6.

13 Weidekamm E, Plozza-Nottebrock H, Forgo I, Dubach UC. Plasma concentrations in pyrimethamine and sulfadoxine and evaluation of pharmacokinetic data by computerized curve fitting. Bull World Health Organ 1982; 60: 115-22.

14 McLeod R, Mack D, Foss R et al. Levels of pyrimethamine in sera and cerebrospinal and ventricular fluids from infants treated for congenital toxoplasmosis. Toxoplasmosis Study Group. Antimicrob Agents Chemother 1992; 36: 1040-8.

15 Hellgren U, Kihamia CM, Bergqvist Y, Lebbad M, Premji Z, Rombo L. Standard and reduced doses of sulfadoxine-pyrimethamine for treatment of Plasmodium falciparum in Tanzania, with determination of drug concentrations and susceptibility in vitro. Trans R Soc Trop Med Hyg 1990; 84: 469-72.
16 Pinon JM, Chemla C, Villena I et al. Early neonatal diagnosis of congenital toxoplasmosis: value of comparative enzyme-linked immunofiltration assay immunological profiles and antiToxoplasma gondii immunoglobulin M (IgM) or IgA immunocapture and implications for postnatal therapeutic strategies. J Clin Microbiol 1996; 34: 579-83.

17 Urien S. MP2 (V 2.0) - a windows application for population pharmacokinetics. In The Population Approach: Measuring and Managing Variability in Response, Concentration, ed Aarons L, Balant LP, Dunhof M, et al. The European Commission (Directorate-General Science, Research and Development) Brussels: 1997; 419-22.

18 Beal SL, Sheiner LB. NONMEM User's Guide. San Francisco: NONMEM Project Group, University of California, 1998.

19 West $\mathrm{GB}$, Brown JH, Enquist BJ. A general model for the origin of allometric scaling laws in biology. Science 1997; 276: 122-6.

20 Anderson BJ, Woollard GA, Holford NH. A model for size and age changes in the pharmacokinetics of paracetamol in neonates, infants and children. Br J Clin Pharmacol 2000; 50: 125-34. 